\title{
Subsolidus phase equilibria and electrical properties of pyrochlores in the $\mathrm{Bi}_{2} \mathrm{O}_{3}-\mathrm{CuO}$ $\mathrm{Ta}_{2} \mathrm{O}_{5}$ ternary system
}

\begin{abstract}
Subsolidus phase relations had been determined for the $\mathrm{Bi}_{2} \mathrm{O}_{3}-\mathrm{CuO}-\mathrm{Ta}_{2} \mathrm{O}_{5}$ (BCT) system over the temperature range $700-950{ }^{\circ} \mathrm{C}$ in air. Two structurally distinct ternary phases of different symmetries, a cubic pyrochlore and a monoclinic zirconolite were observed. Phasepure pyrochlores existed in a unique boomerang-shaped area, which could be described using the general formula, $\mathrm{Bi}_{2.48+y} \mathrm{Cu}_{1.92-\mathrm{x}} \mathrm{Ta}_{3.6+\mathrm{x}-\mathrm{y}} \mathrm{O}_{14.64+3 \mathrm{x} / 2-\mathrm{y}}: 0.00(1) \leq \mathrm{x} \leq 0.80(1)$ and 0.00 (1) $\leq \mathrm{y} \leq 0.60(1)$, respectively. BCT subsolidus pyrochlores exhibited low electrical conductivities, $10^{-7}-10^{-6} \mathrm{~S} \mathrm{~cm}^{-1}$, moderate dielectric constants, $\varepsilon^{\prime}, \sim 60-80$ and low dielectric losses, $\tan \delta, \sim 0.01-0.20$ at $1 \mathrm{MHz}$ and ambient temperature, $\sim 28{ }^{\circ} \mathrm{C}$. The recorded low activation energies, $0.32-0.40 \mathrm{eV}$ suggesting these electroceramics were of semiconductortype at elevated temperatures.
\end{abstract}

Keyword: Ternary phase diagram; X-ray methods; Electrical conductivity; Tantalates 